\title{
Synaptopodin regulates the actin-bundling activity of $\alpha$-actinin in an isoform-specific manner
}

\author{
Katsuhiko Asanuma, ${ }^{1}$ Kwanghee Kim, ${ }^{1}$ Jun Oh, ${ }^{1}$ Laura Giardino, ${ }^{1}$ Sophie Chabanis, ${ }^{2}$ \\ Christian Faul, ${ }^{1,3}$ Jochen Reiser, ${ }^{1}$ and Peter Mundel1,3

\begin{abstract}
1Department of Medicine, Albert Einstein College of Medicine, New York, New York, USA. ${ }^{2}$ Structural and Computational Biology Unit, European Molecular Biology Laboratory, Heidelberg, Germany. 3Department of Anatomy and Structural Biology, Albert Einstein College of Medicine, New York, New York, USA.
\end{abstract}

\begin{abstract}
Synaptopodin is the founding member of a novel class of proline-rich actin-associated proteins highly expressed in telencephalic dendrites and renal podocytes. Synaptopodin-deficient $\left(\operatorname{synpo}^{-/}\right)$mice lack the dendritic spine apparatus and display impaired activity-dependent long-term synaptic plasticity. In contrast, the ultrastructure of podocytes in synpo ${ }^{-/-}$mice is normal. Here we show that synpo-/- mice display impaired recovery from protamine sulfate-induced podocyte foot process (FP) effacement and LPS-induced nephrotic syndrome. Similarly, $\operatorname{synpo}^{-/-}$podocytes show impaired actin filament reformation in vitro. We further demonstrate that synaptopodin exists in 3 isoforms, neuronal Synpo-short (685 AA), renal Synpo-long (903 AA), and Synpo-T (181 AA). The $\mathrm{C}$ terminus of Synpo-long is identical to that of Synpo-T. All 3 isoforms specifically interact with $\alpha$-actinin and elongate $\alpha$-actinin-induced actin filaments. syn $\mathrm{po}^{-/-}$mice lack Synpo-short and Synpo-long expression but show an upregulation of Synpo-T protein expression in podocytes, though not in the brain. Gene silencing of Synpo-T abrogates stress-fiber formation in synpo ${ }^{-/-}$podocytes, demonstrating that Synpo-T serves as a backup for Synpo-long in synpo-/- podocytes. In concert, synaptopodin regulates the actin-bundling activity of $\alpha$-actinin in highly dynamic cell compartments, such as podocyte FPs and the dendritic spine apparatus.
\end{abstract}

\section{Introduction}

Originally we identified synaptopodin as an actin-associated protein of differentiated podocytes, where it is part of the actin-based contractile apparatus in the foot processes (FPs) (1). Synaptopodin is the founding member of a novel class of actin-associated proteins (2) without significant homology to any known protein except myopodin, the second member of the gene family (3). Synaptopodin is also expressed in the brain, where it is found at the postsynaptic density (2) and the spine apparatus in a subset of telencephalic neurons $(2,4)$. Analogous to the kidney (1), synaptopodin gene expression in the brain and in cultured hippocampal neurons is differentiation-dependent (2). These results and experiments with cultured cells suggested that synaptopodin plays a role in modulating the actin-based shape and motility of dendritic spines and podocyte FPs (2). To determine the in vivo function of synaptopodin, we targeted the murine synaptopodin gene by homologous recombination in ES cells (5). synpo- ${ }^{-1}$ mice are viable and fertile, but their hippocampal neurons are devoid of the spine apparatus and they show impaired activity-dependent long-term synaptic plasticity (5).

$\alpha$-Actinin is a ubiquitously expressed protein that is regarded as the ancestral molecule within a family of actin-binding proteins comprising spectrin, dystrophin, and utrophin $(6,7)$. Muscle and nonmuscle isoforms of $\alpha$-actinin have been previously described $(6,8)$.

Nonstandard abbreviations used: FP, foot process; FSGS, focal segmental glomerulosclerosis; GBM, glomerular basement membrane; KLH, keyhole limpet hemocyanin; ORF, open reading frame; PS, protamine sulfate; SD, slit diaphragm; siRNA, small interfering RNA; synpo ${ }^{-/}$, synaptopodin-deficient; TEM, transmission electron microscopy.

Conflict of interest: The authors have declared that no conflict of interest exists.

Citation for this article: J. Clin. Invest. 115:1188-1198 (2005).

doi:10.1172/JCI200523371
The $4 \alpha$-actinin genes encode highly homologous proteins that form head-to-tail homodimers (9). The best-defined function of $\alpha$-actinin is to cross-link and bundle actin filaments, but it also interacts with a large and diverse set of other proteins (10). In skeletal and cardiac muscle, $\alpha$-actinin is found at the $\mathrm{Z}$ disk, where it cross-links anti-parallel actin filaments from adjacent sarcomeres. In nonmuscle cells, $\alpha$-actinin is involved in the organization of the cortical cytoskeleton and is also found along stress fibers and in focal contacts, where it interacts with a variety of cytoskeletal and membrane-associated proteins (10). Disorders of the actin cytoskeleton result in distinct diseases, and point mutations in the $\alpha$-actinin-4 (ACTN4) gene lead to an autosomal dominant form of human focal segmental glomerulosclerosis (FSGS) (11) that seems to be caused by the aggregation and rapid degradation of $\alpha$-actinin-4 (12).

Here, we report that synpo-/- mice develop a time-dependent downregulation of $\alpha$-actinin-2 expression in the brain but not of $\alpha$-actinin-4 expression in the kidney. We also show that synpo-/podocytes display a delayed re-formation of podocyte actin filaments in vivo and in vitro. We further show that synaptopodin specifically interacts with $\alpha$-actinin and that this interaction is functionally significant because synaptopodin bundles and elongates $\alpha$-actinin-induced actin filaments in an isoform-specific manner. Finally, we demonstrate that the impaired plasticity of the podocyte actin cytoskeleton in synpo-/- mice is due to the partial rescue of synaptopodin function by Synpo-T, a truncated isoform that is upregulated in synpo-/- podocytes.

\section{Results}

Impaired recovery of synpo-/- mice from FP effacement induced by protamine sulfate. The brains of synpo-/- mice lack a dendritic spine apparatus (5). In contrast, the kidney structure did not reveal any 
A

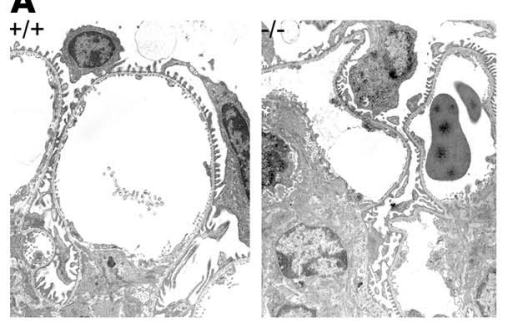

B

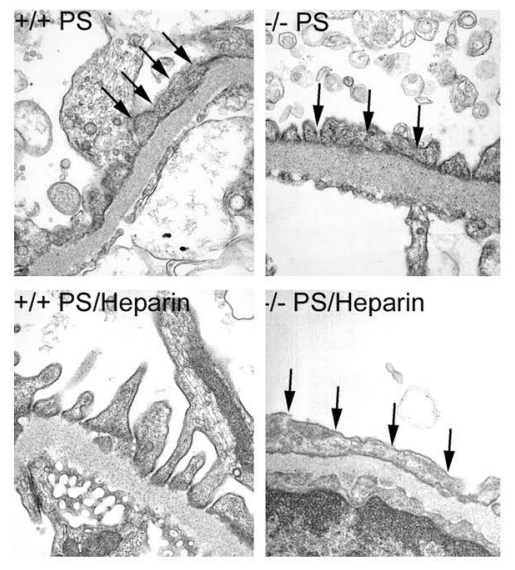

C

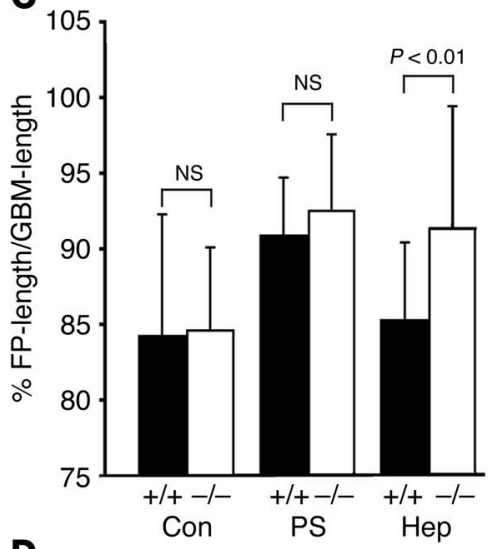

D

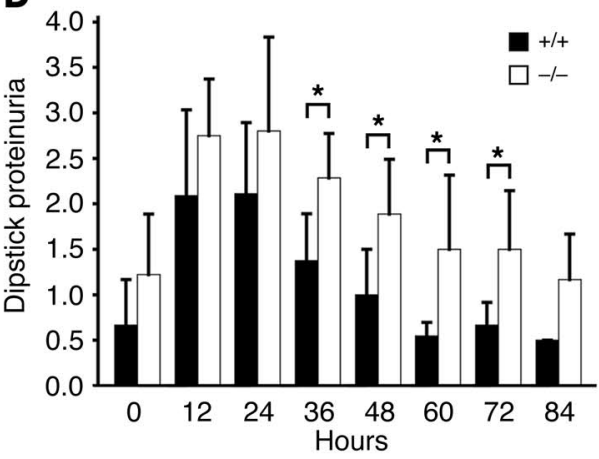

E

$\mathbf{F}$
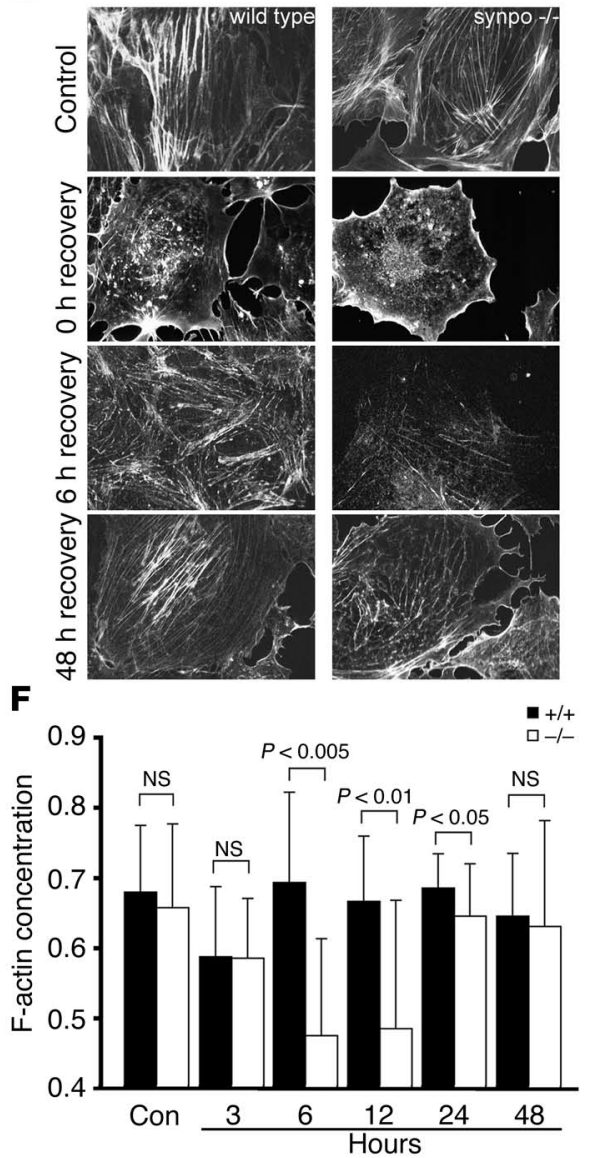

Figure 1

Delayed actin filament reformation in synpo-/- podocytes. (A) By TEM analysis, podocytes of synpo-/- mice appear structurally normal (magnification, $\times 20,000) .+/+$, wild-type; -/-, synpo ${ }^{-/-}$. (B) Impaired recovery of synpo-/- mice from PS-induced FP effacement. Top: comparable degrees of effacement (arrows) in wild-type and synpo-/- mice. Bottom: complete recovery after heparin treatment in wild-type but not synpo-/- mice (magnification, $\times 40,000$ ). (C) Quantitative analysis showing significantly delayed FP re-formation in synpo ${ }^{-/-}$mice during recovery induced by heparin sulfate (Hep) $(P<0.01)$. Con, control. (D) LPS injection causes delayed recovery from proteinuria in synpo ${ }^{-/-}$mice. Statistically significant changes were found between 36 and 72 hours; ${ }^{*} P<0.05$. (E) Confocal imaging showing delayed re-formation of stress fibers in synpo ${ }^{-/-}$podocytes (magnification, $\times 600$ ). No changes were found between wild-type and synpo-/- podocytes before and directly after actin depolymerization with cytochalasin D (0 hours recovery). After washout of cytochalasin D, actin fibers were fully reestablished in wild-type cells after 6 hours. In synpo ${ }^{-/}$podocytes, stress fiber formation did not return to control levels before 48 hours. (F) Quantitative analysis of F-actin content in wild-type and synpo-/- podocytes. No significant differences were found in untreated controls and at 3 hours after removal of cytochalasin D. Wild-type cells returned to control levels at 6 hours. In contrast, synpo-/- cells displayed significantly reduced F-actin content at 6,12 , and 24 hours after washout. At 48 hours, synpo-/- mice displayed wild-type level.

obvious differences between podocytes of wild-type and synpo-/mice (Figure 1A). The absence of an overtly abnormal kidney phenotype prompted us to test whether synpo-/- mice display impaired plasticity of the podocyte actin cytoskeleton, which would become overt during the dynamic reorganization of the actin cytoskeleton. We assessed the dynamics of the podocyte actin cytoskeleton of wild-type and synpo-/- mice using the protamine sulfate (PS) model of acute FP effacement (13). PS caused comparable degrees of FP effacement in both groups (Figure 1B, top, arrows). However, after reperfusion with heparin, which neutralizes the activity of PS (13), the normal FP architecture was completely restored in wildtype mice (Figure 1B, bottom left) but not in synpo- $\mathrm{o}^{-/}$littermates (Figure 1B, bottom right, arrows). To quantify the differences between wild type and synpo- $0^{-1-}$ mice, we determined the percentage of glomerular basement membrane (GBM) covered by FP (Figure 1C). Under normal conditions in both wild-type and synpo- mice, approximately $85 \%$ of the outer GBM surface is covered by podocyte FPs, whereas approximately $15 \%$ is covered by the slit diaphragm (SD) (percentage of GBM covered by FPs, $84.2 \% \pm 4.9 \%$ in wildtype versus $84.6 \% \pm 3.0 \%$ in synpo $0^{-/-}$mice; $P=\mathrm{NS}$, Student's $t$ test). During FP effacement, most of the GBM area is plastered by fused podocyte processes $(90.9 \% \pm 2.2 \%$ in wild-type versus $92.5 \% \pm 2.4 \%$ in synpo $0^{-/}$mice; $P=\mathrm{NS}$, Student's $t$ test), thereby reducing the area covered by the SD to $9 \%$ in wild-type and $7.5 \%$ in synpo- ${ }^{-1-}$ mice. In other words, PS leads to a $40 \%$ reduction of the SD area available for glomerular filtration in wild-type mice versus a $50 \%$ reduction of SD area in synpo-/- mice. After reperfusion with heparin, wild-type mice returned to baseline levels, whereas synpo-/- mice 


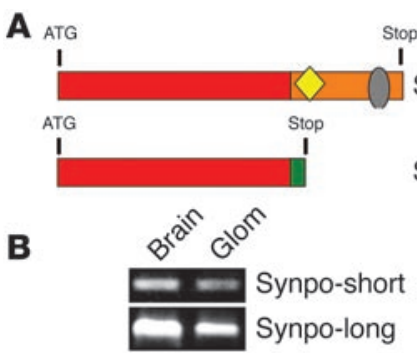

Synpo-long protein (903 AA)

Synpo-short protein (685 AA)

D

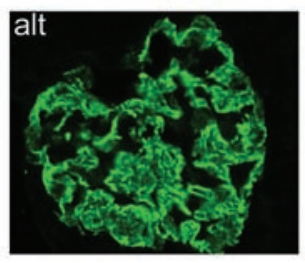

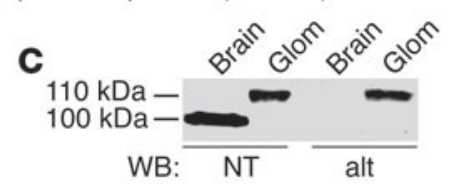

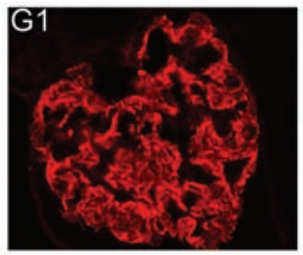

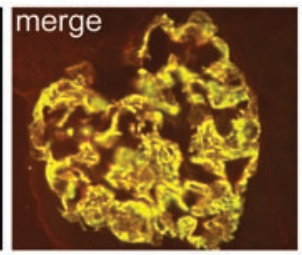

\section{Figure 2}

Synaptopodin isoform expression in kidney and brain. (A) Comparison of Synpo-long and Synpo-short. The red box shows the first 670 AA that are shared between both isoforms; the orange box shows AA 671-903 corresponding to the Synpo-alt fragment of Synpo-long. Synpo-alt contains a LPPPP motif (yellow) for ENA/VASP binding and a PPRPF motif (gray) for homer binding. (B) RT-PCR with isoform-specific primers reveals the expression of both Synpo-short and Synpo-long mRNA in brain and kidney glomeruli (Glom). (C) Western blotting (WB) with the polyclonal NT antibody against an epitope present in both Synpo-long and Synpo-short and the Synpo-alt-specific antibody (alt). At the protein level, only Synpo-long is expressed in the kidney, whereas only Synpo-short is expressed in the brain. (D) Double-labeling immunofluorescence microcopy of Synpo-alt with the pan-synaptopodin monoclonal antibody G1 confirmed the expression of Synpo-long in podocytes (magnification, x650). remained effaced (percentage of GBM covered by FPs, $85.2 \% \pm 1.1 \%$ in wild-type versus $91.3 \% \pm 2.9 \%$ in synpo-/- mice; $P<0.01$, Student's $t$ test). Hence, in wild-type mice, approximately $15 \%$ of the GBM is covered again by SD, similar to control levels. In contrast, synpo $0^{-/-}$mice remain effaced, with only $8.7 \%$ of the GBM covered by the $\mathrm{SD}$, representing a $42 \%$ reduction. These data reflect an almost complete persistence of FP effacement in synpo- $\mathrm{o}^{-/}$mice that is consistent with a severe recovery defect.

Delayed recovery of synpo-/- mice from LPS-induced proteinuria. Due to the experimental setup, the PS/heparin model terminates with the death of the animal after 60 minutes at the end of the experiments $(13,14)$. Hence, it cannot be used to determine whether recovery of synpo-/- mice does not occur at all or is only delayed compared with wild-type mice. Therefore, we used the LPS model of transient nephrotic syndrome, in which FP effacement and massive proteinuria of wild-type mice develop within 12-24 hours and return to baseline levels within 72 hours (15). The time course of proteinuria was measured in wild-type and synpo-/- mice at various time points before (0 hours) and after (12, 24, 36, 48, 60, 72, and 84 hours) injection of LPS (Figure 1D). Urine protein was markedly increased in synpo- $\mathrm{o}^{-/}$mice at all time points examined, with highly statistically significant differences at 36,48 , and 72 hours. At 36 hours of observation, proteinuria levels started to decline in both groups but remained significantly higher in synpo ${ }^{-/-}$mice ( $1.37 \pm 0.52$ in wild-type versus $2.29 \pm 0.49$ dipstick proteinuria in synpo-/- mice; $P<0.005)$. At 48 hours of observation, proteinuria had returned to baseline values in wild-type mice but remained significantly elevated in synpo ${ }^{-/-}$mice (1.00 \pm 0.50 in wild-type versus $1.8 \pm 0.60$ dipstick proteinuria in synpo-/- mice; $P<0.005)$. Proteinuria continued to be elevated in synpo-/- mice at 60 hours $(0.55 \pm 0.15$ in wild-type versus $1.5 \pm 0.82$ dipstick proteinuria in synpo-/- mice; $P<0.005)$ and 72 hours $(0.62 \pm 0.25$ in wild-type versus $1.5 \pm 0.65$ dipstick proteinuria in synpo-/- mice; $P<0.005$ ) and returned to baseline at 84 hours (Figure 1D), which again was not different between both groups.

Delayed stress fiber re-formation in synpo- ${ }^{-/}$podocytes. Next, we analyzed the actin cytoskeleton of wild-type and synpo-/- cultured podocytes before and during recovery from cytochalasin Dinduced actin fiber disruption (Figure 1E). No differences in actin fiber numbers were observed between untreated wild-type and synpo-/- podocytes or immediately after depolymerization of the actin cytoskeleton (Figure 1E). However, after washout of cytochalasin D during the recovery of the actin cytoskeleton, a clear difference was observed (Figure 1E). In wild-type cells, actin stress fibers were fully reestablished by 6 hours (Figure 1E). In contrast, in synpo-/- cells, stress fiber re-formation was greatly impaired after 6 hours after washout, and control levels were not reached before 48 hours (Figure 1E). To analyze these data in a quantitative fashion, we used confocal microscopy and measured the F-actin concentration of wild-type and synpo-/- cells (Figure 1F). There was no significant difference in the F-actin content before or 3 hours after washout of cytochalasin D (Figure 1F). Wild-type cells reached the baseline concentration after 6 hours. In contrast, the F-actin concentration was decreased in synpo-/- cells at 6 hours $(0.69 \pm 0.12$ in wild-type versus $0.47 \pm 0.14$ pixels/cell volume in synpo-/- cells; $P<0.005), 12$ hours $(0.67 \pm 0.10$ in wild-type versus $0.49 \pm 0.18$ pixels/cell volume in synpo ${ }^{-/-}$cells; $P<0.01)$, and 24 hours $(0.69 \pm 0.05$ in wild-type versus $0.65 \pm 0.08$ pixels/cell volume in synpo $0^{-/-}$cells; $\left.P<0.05\right)$. After 48 hours, synpo $0^{-/-}$ cells reached an F-actin concentration that was comparable to wild-type levels $(0.65 \pm 0.1$ in wild-type versus $0.63 \pm 0.15$ pixels/ cell volume in synpo-/- cells; $P=\mathrm{NS}$ ). Taken together, these data show that the lack of synaptopodin delays the re-formation of the podocyte actin cytoskeleton in vivo and in vitro. At this point 2 important questions arise: why do synpo-/- null mice show an overtly abnormal phenotype in the brain (5) but not in the kidney, and what is the molecular function of synaptopodin? The remainder of this paper is focused on answering these questions.

Synpo-long protein is expressed in podocytes but not in the brain. The originally described human Synpo-short version encodes a protein with $685 \mathrm{AA}(2)$. Of these, the first $670 \mathrm{AA}$ are identical with the novel Synpo-long isoform, which encodes a 903-AA protein (GenBank accession number AB028952) (Figure 2A). The $\mathrm{C}$ terminus of Synpo-long contains a 234-AA fragment (termed Synpo-alt) that is not present in Synpo-short. The Synpo-alt fragment is proline rich and contains consensus motifs for Ena/VASP binding (LPPPP) and homer binding (PPRPF) (16). By RT-PCR with isoform-specific primers, mRNA expression of both Synposhort and Synpo-long was observed in mouse brain and isolated glomeruli (Figure 2B). The previously described polyclonal NT antibody (2) is directed against an epitope shared by Synpo-short and Synpo-long, and it detected the previously described 110-kDa 


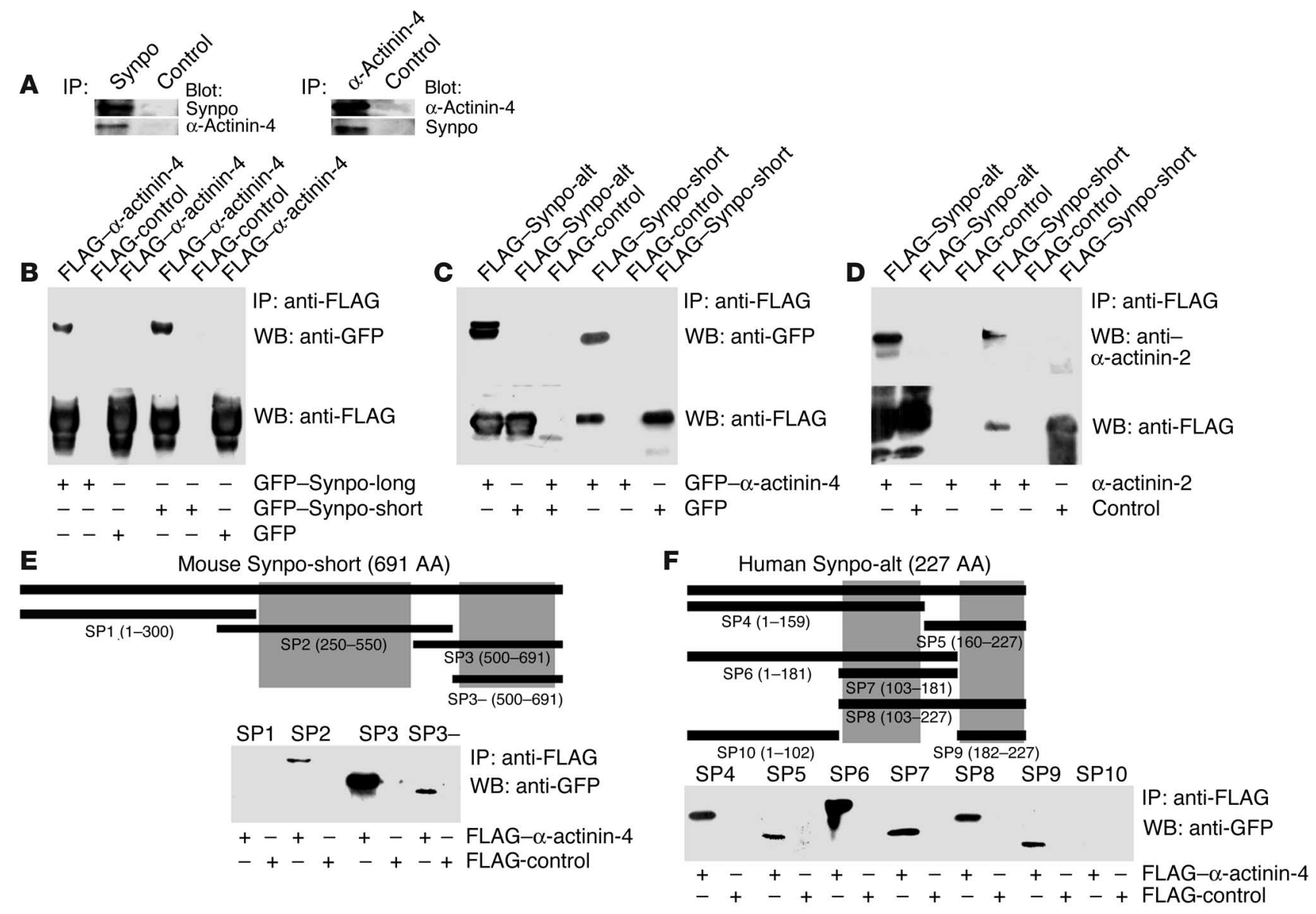

Figure 3

Synpo-short and Synpo-long interact with $\alpha$-actinin-4 and $\alpha$-actinin-2. (A) Coimmunoprecipitation experiments showing that endogenous synaptopodin interacts with $\alpha$-actinin-4 in podocytes. Left: Immunoprecipitation with anti-synaptopodin (Synpo). Right: Immunoprecipitation with anti- $\alpha$-actinin-4. No interaction was found with a control antibody. (B) GFP-tagged Synpo-long and Synpo-short coimmmunoprecipitated with FLAG-tagged $\alpha$-actinin-4 but not with the FLAG control. (C) In a converse experiment, GFP-tagged $\alpha$-actinin-4 coprecipitated with FLAG-Synpoalt and FLAG-Synpo-short. No interaction was found with the GFP control. (D) FLAG-Synpo-alt and FLAG-Synpo-short also interact with GFP-tagged $\alpha$-actinin-2. (E) Synpo-short contains 2 independent nonoverlapping interaction sites (gray boxes) for $\alpha$-actinin-4 that are contained in AA 300-550 and 550-691. In contrast, the SP1 fragment and the FLAG control do not interact with $\alpha$-actinin. (F) Synpo-alt also contains 2 independent nonoverlapping $\alpha$-actinin interaction sites that are localized in AA 103-159 and 182-227 of the Synpo-alt fragment. Hence, Synpolong contains $4 \alpha$-actinin interacting sites, whereas Synpo-short contains only $2 \alpha$-actinin interacting sites.

band in cytosolic fractions from isolated glomeruli and the $100-\mathrm{kDa}$ band in the brains of adult wild-type mice (Figure 2C, left). Therefore, we generated a peptide antibody specific to Synpolong and Synpo-alt, which detected the 110-kDa band in glomeruli but not in the brains of wild-type mice (Figure 2C, right). By immunofluorescence microscopy of adult mouse kidney, Synpolong was detected in podocytes (Figure 2D), where it colocalized with the previously raised monoclonal antibody G1 that recognizes both the 110 - and $100-\mathrm{kDa}$ isoforms (2). In contrast, Synpo-long was not detected by immunofluorescence in mouse brain (data not shown).

Synpo-long and Synpo-short interact with $\alpha$-actinin- 2 and $\alpha$-actinin-4. In an effort to delineate isoform-specific differences in synaptopodin function, a human kidney cDNA library was screened using the yeast 2 -hybrid system. To identify proteins interacting specifically with Synpo-long, the Synpo-alt fragment (AA 671-903) (Figure 2A) was fused to the DNA binding domain of GAL4. Among several others, we found 52 cDNA clones encoding $\alpha$-actinin- 4 and 6 clones encoding $\alpha$-actinin- 1 . $\alpha$-Actinin- 4 -mediated FSGS is an inherited kidney disease that seems to be caused by the aggregation and rapid degradation of $\alpha$-actinin-4 $(11,12)$. Therefore, we focused on characterizing the functional significance of the synaptopodin- $\alpha$-actinin- 4 interaction in more detail. To confirm the interaction between synaptopodin and $\alpha$-actinin- 4 , endogenous proteins were immunoprecipitated from podocyte extracts using the Synpo-alt antibody and a polyclonal antibody against $\alpha$-actinin- 4 (11). Anti-Synpo-alt precipitated Synpo-long from the extract and also coprecipitated $\alpha$-actinin- 4 (Figure 3A, left). Conversely, anti$\alpha$-actinin-4 coimmunoprecipitated Synpo-long (Figure 3A, right). No interaction was found with an irrelevant control antibody (Figure 3A). Next, FLAG- $\alpha$-actinin-4 was coexpressed in HEK293 cells with GFP-Synpo-long or GFP-Synpo-short; both coprecipitated with FLAG- $\alpha$-actinin-4 (Figure 3B). In a complementary experiment, GFP- $\alpha$-actinin- 4 coprecipitated with FLAG-Synpo- 

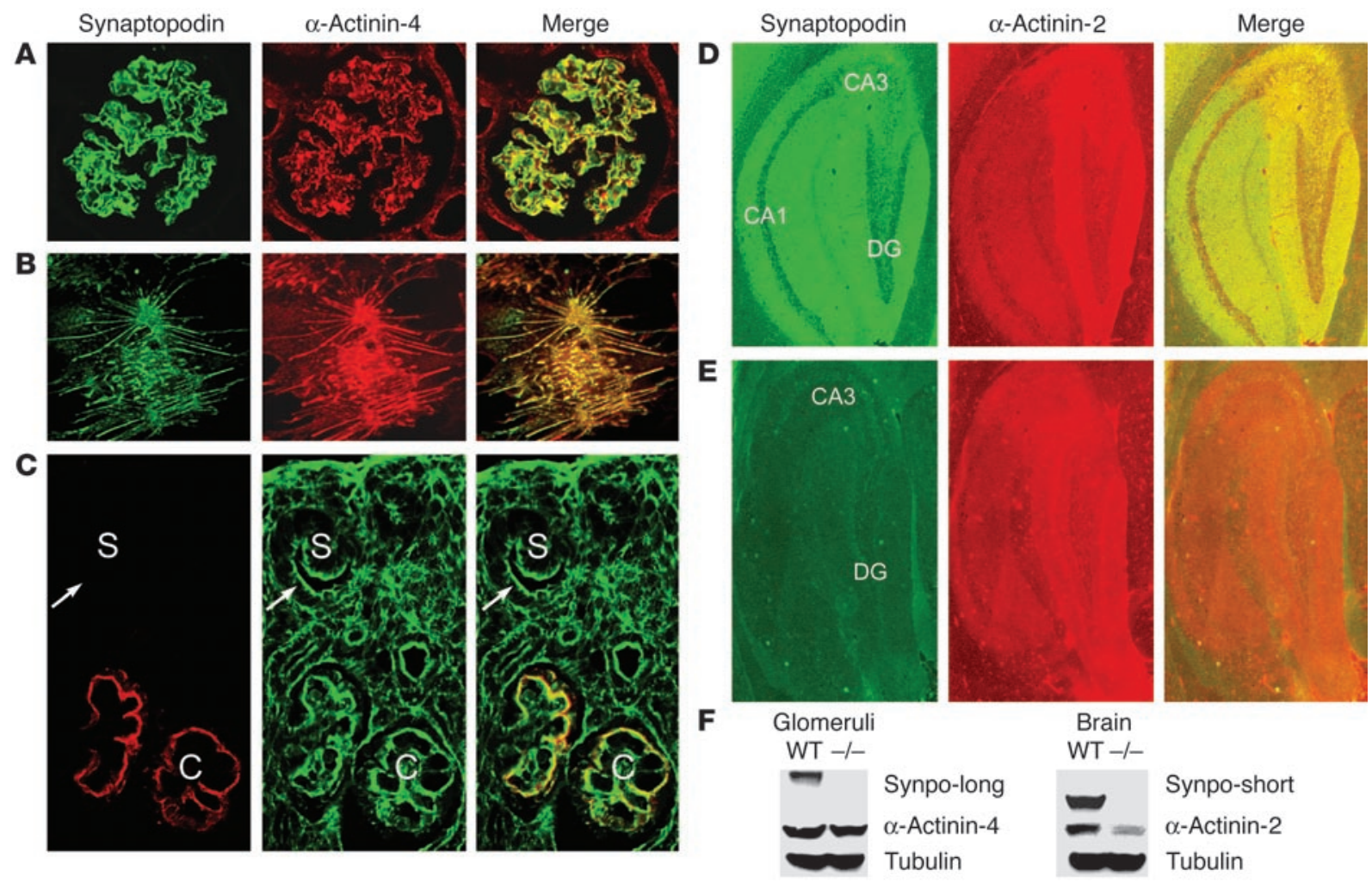

\section{Figure 4}

Synaptopodin colocalizes with $\alpha$-actinin in tissues and cultured cells. (A) In the adult kidney, synaptopodin colocalizes with $\alpha$-actinin-4 in podocytes (magnification, $\times 650$ ). (B) Synaptopodin colocalizes with $\alpha$-actinin-4 along actin filaments in differentiated cultured wild-type podocytes (magnification, $\times 650$ ). (C) During kidney development, $\alpha$-actinin-4 is already found in undifferentiated podocytes (arrows) of the S-shaped body stage (S) that have a cortical actin cytoskeleton. In contrast, synaptopodin is first found in the capillary loop stage (C) when podocytes start to develop long, unbranched contractile actin bundles (magnification, $\times 450$ ). (D) Codistribution of synaptopodin with $\alpha$-actinin-2 in the CA1, CA3, and dendate gyrus (DG) regions in the hippocampus of a 6-month-old wild-type mouse (magnification, $\times 650$ ). (E) Lack of synaptopodin and downregulation of $\alpha$-actinin-2 labeling in all regions of the hippocampus in an age-matched synpo-/- mouse (magnification, $\times 650)$. (F) Western blot analysis of cytosolic extract from glomeruli (left) and brains (right) of 6-month-old wild-type and synpo-/- mice. The levels of $\alpha$-actinin-4 are not different between wild-type and synpo-/- mice. In contrast, there is a strong downregulation of $\alpha$-actinin- 2 in the brains of synpo ${ }^{-/-}$mice. Equal protein loading was confirmed by reprobing for tubulin.

alt and FLAG-Synpo-short (Figure 3C). In both cases, GFP alone did not interact with FLAG- $\alpha$-actinin- 4 or FLAG-synaptopodin and, therefore, served as a negative control (Figure 3, B and C). In the brain, synaptopodin $(2,5)$ and $\alpha$-actinin-2 (17) are associated with the dendritic spine apparatus. Therefore, we tested whether Synpo-alt or Synpo-short would also interact with $\alpha$-actinin-2. GFP- $\alpha$-actinin-2 specifically coprecipitated with FLAG-Synpo-alt and FLAG-Synpo-short (Figure 3D). To map the synaptopodin$\alpha$-actinin-4 interaction sites, various FLAG-Synpo-short (Figure 3E) and FLAG-Synpo-alt (Figure 3F) truncation constructs were coexpressed with GFP- $\alpha$-actinin- 4 in HEK293 cells and tested for their ability to coprecipitate with GFP- $\alpha$-actinin- 4 . As described above, GFP alone served as negative control. With this approach, 2 separate $\alpha$-actinin binding sites were found in Synpo-short spanning AAs 300-550 and 550-691 (Figure 3E, gray boxes). These $\alpha$-actinin binding sites are shared by Synpo-long. In addition, Synpo-long harbors 2 additional $\alpha$-actinin binding sites not found in Synpo-short that comprise AAs 103-159 and 182-227 of the Synpo-alt fragment (Figure 3F, gray boxes). Hence, Synpoalt and Synpo-short each contain at least 2 independent binding sites for $\alpha$-actinin.
Synaptopodin colocalizes with and modulates the expression of $\alpha$-actinin. The spatial and temporal relationship of synaptopodin and $\alpha$-actinin isoforms was analyzed by immunofluorescence microscopy and Western blotting in wild-type and synpo-/- mice. In the adult mouse kidney, Synpo-long colocalized with $\alpha$-actinin- 4 in podocytes (Figure 4A). Similarly, in differentiated cultured podocytes, synaptopodin colocalized with $\alpha$-actinin in a dotted pattern along stress fibers (Figure 4B). During kidney development, the expression of $\alpha$-actinin- 4 in podocytes preceded the expression of synaptopodin (Figure 4C). $\alpha$-Actinin-4 was first expressed during the S-shaped body stage of glomerular development in which the presumptive podocytes display characteristics of epithelial cells, including apically located tight junctions and a cortical actin cytoskeleton (18). In contrast, synaptopodin was first detected in the subsequent capillary loop stage (Figure 4C) during which podocytes start developing their characteristic FP harboring an actin-based contractile system comparable to that of smooth muscle cells (19). These data suggest a role for synaptopodin in the developmental reorganization of the $\alpha$-actinin-4-based podocyte actin cytoskeleton (see below).

In the hippocampus of wild-type mice, synaptopodin colocalized with $\alpha$-actinin-2 (Figure 4D). In synpo-/- mice, hippocampal 

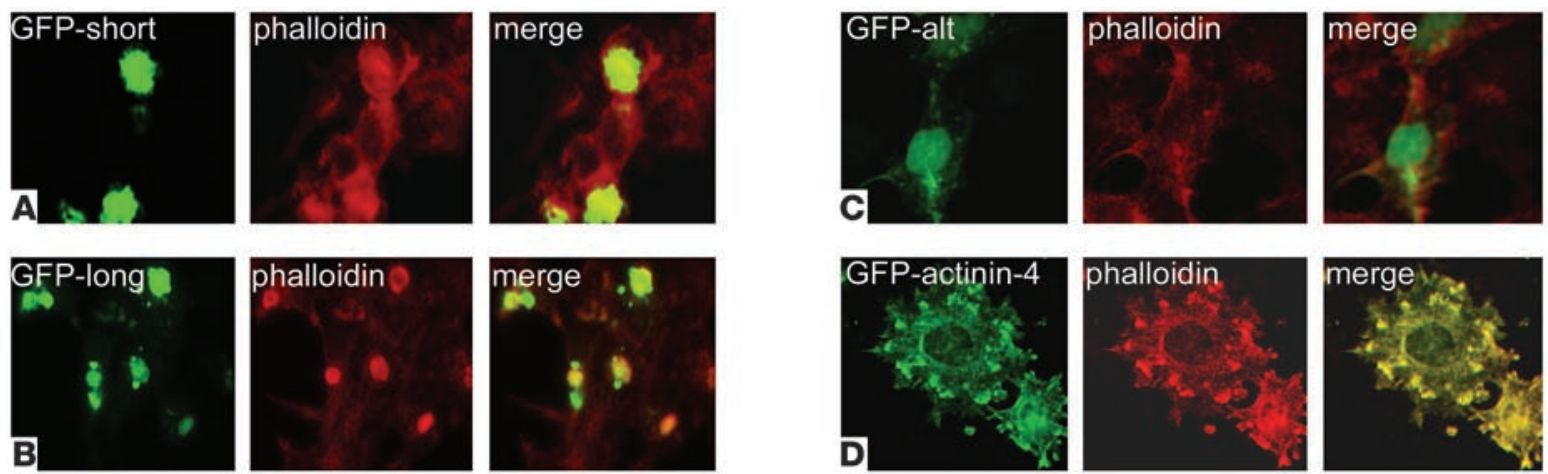

GFP-short + FLAG- $\alpha$-actinin-4
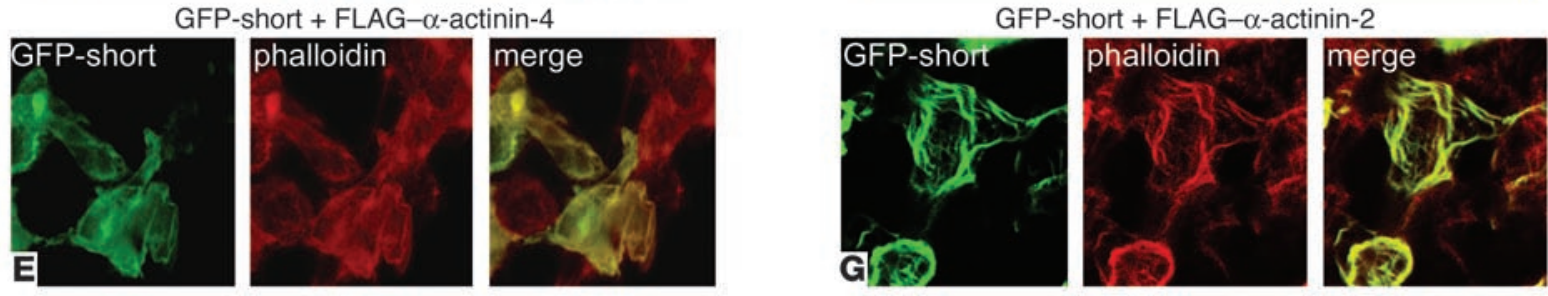

GFP-alt + FLAG- $\alpha$-actinin-4
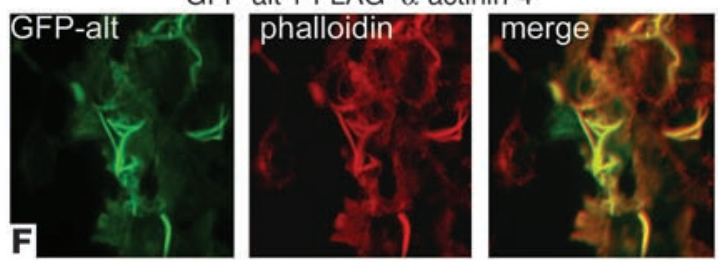

GFP-alt + FLAG- $\alpha$-actinin-2
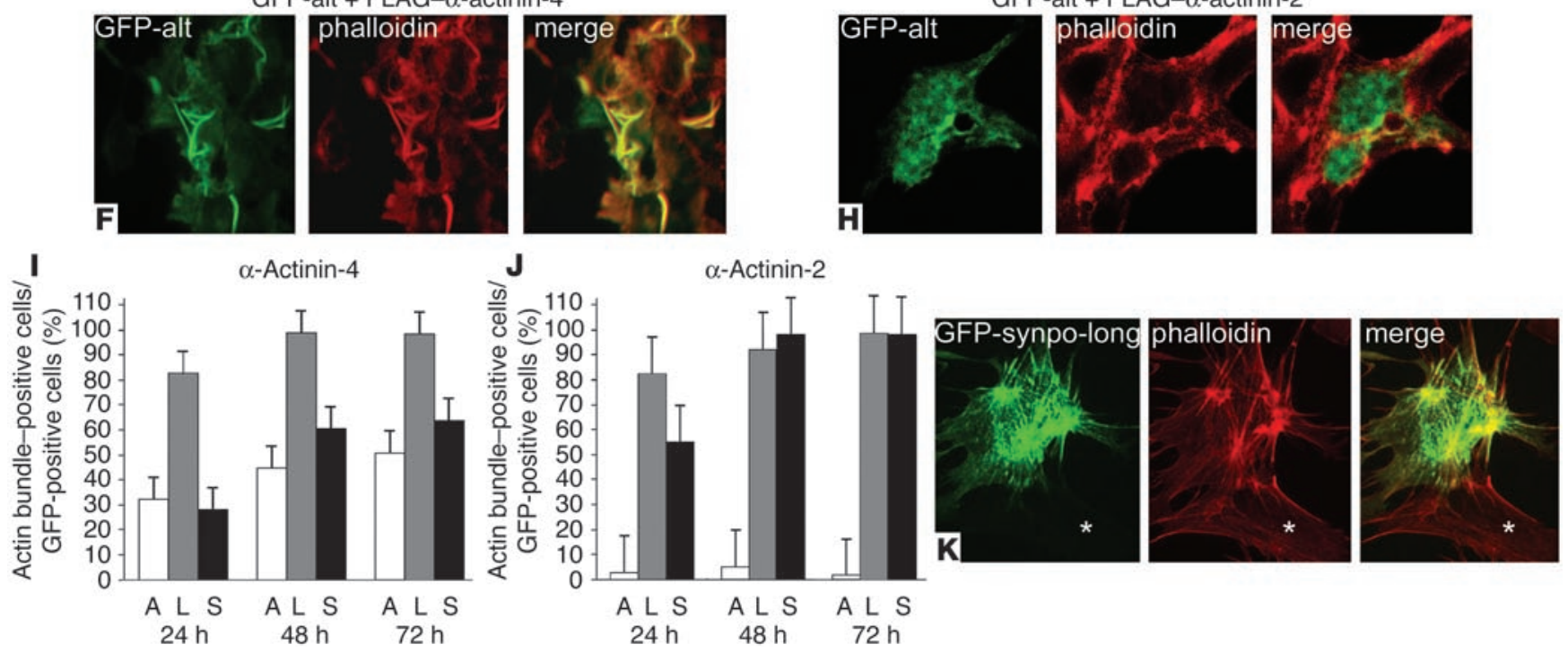

Figure 5

Synaptopodin cooperates with $\alpha$-actinin in the elongation and bundling of $\alpha$-actinin-induced actin filaments. (A) GFP-Synpo-short (GFP-short) or (B) GFP-Synpo-long (GFP-long) induce and colocalize with amorphous cytoplasmic phalloidin-positive aggregates, suggesting the presence of an actin binding site. (C) GFP-Synpo-alt (GFP-alt) does not induce phalloidin-positive aggregates, showing that the actin binding site is localized in the first 670 AA that are shared between Synpo-long and Synpo-short. (D) Single transfection of $\alpha$-actinin-4 induces short, branched actin filaments. (E) Cotransfection of GFP-Synpo-short with FLAG- $\alpha$-actinin-4 converts the short, branched filaments into long, parallel, unbranched actin bundles. (F) Inhibition of actin-branching activity of FLAG- $\alpha$-actinin-4 is also observed after cotransfection with GFP-Synpoalt. (G) Cotransfection of GFP-Synpo-short with FLAG- $\alpha$-actinin-2 also induces long, unbranched actin bundles. (H) In contrast, cotransfection of GFP-Synpo-alt with FLAG- $\alpha$-actinin-2 does not affect $\alpha$-actinin-2-induced short, branched cortical actin filaments. (I and $\mathbf{J}$ ) Quantitative analysis of actin-bundling kinetics. The data are presented as percentage of actin bundle-positive cells per GFP-positive cells. (I) Cells coexpressing $\alpha$-actinin-4 and GFP-Synpo-long (L) show faster kinetics of actin bundling at 24, 48, and 72 hours than cells expressing GPF-Synpo-alt (A) or GFP-Synpo-short (S). (J). After cotransfection with $\alpha$-actinin-2, GFP-Synpo-long and GFP-Synpo-short show comparable kinetics, whereas virtually no actin-bundling activity is detected at any time points in cells coexpressing $\alpha$-actinin-2 and GFP-Synpo-alt. (K) Transfection of undifferentiated wild-type podocytes with GFP-Synpo-long induces stress fibers similar to those found in differentiated wild-type cells (see Figure 4B). No stress fibers are found in nontransfected cells (asterisk). Magnification, $\times 600(\mathbf{A}-\mathbf{H}) ; \times 450(\mathbf{K})$.

immunolabeling for synaptopodin was absent, as shown before (5). Of note, there was a downregulation of $\alpha$-actinin-2 in 6-month-old synpo-/- mice (Figure 4E) compared with wild-type littermates (Figure 4D). Next, we assessed the expression of synaptopodin and $\alpha$-actinin by Western blot analysis of glomerular and forebrain extracts from 2- (data not shown) and 6-month-old (Figure 4F) synpo-/- mice. Synpo-long was expressed in glomeruli of wildtype but not synpo-/- mice. Similarly, Synpo-short was expressed 
A

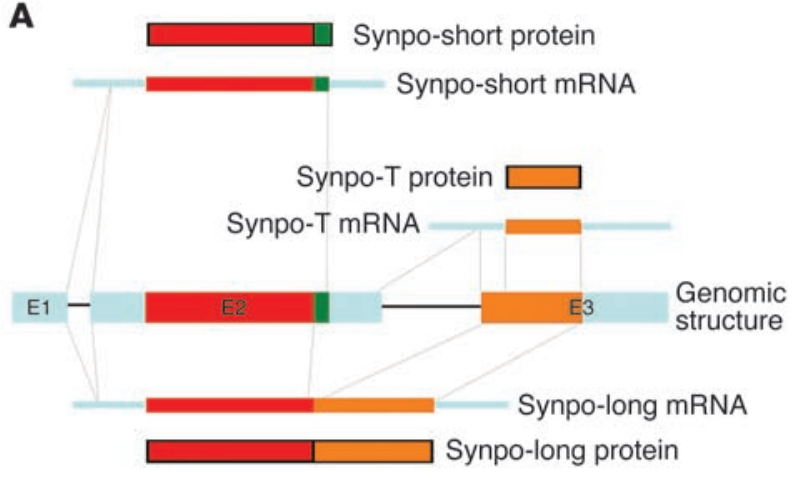

B

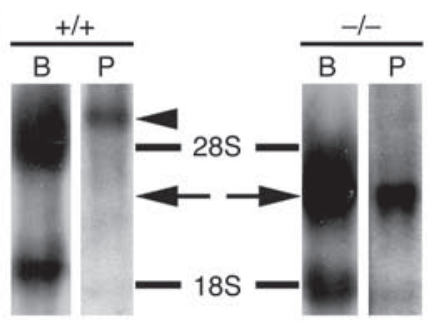

D

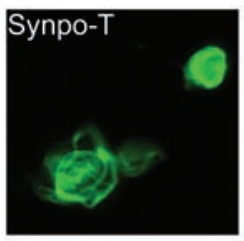

C
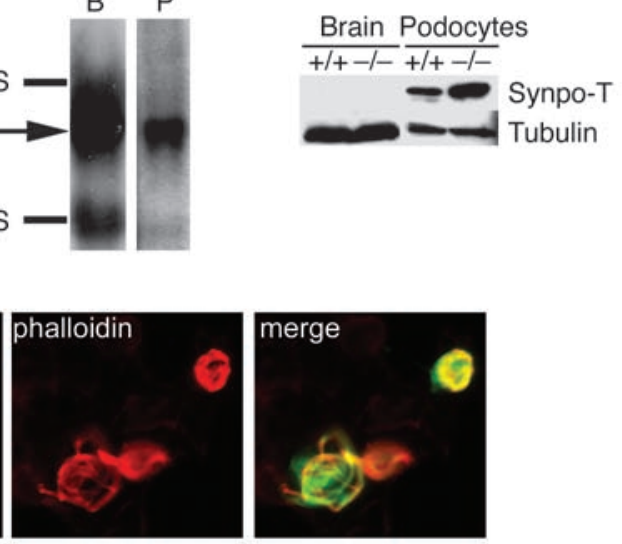

E

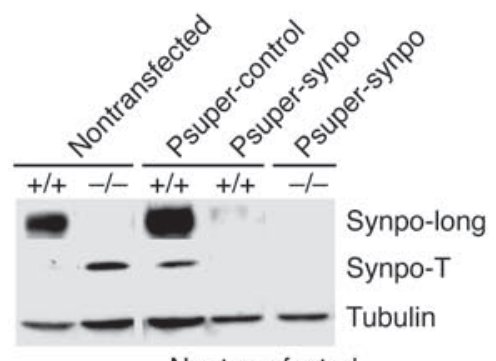

F

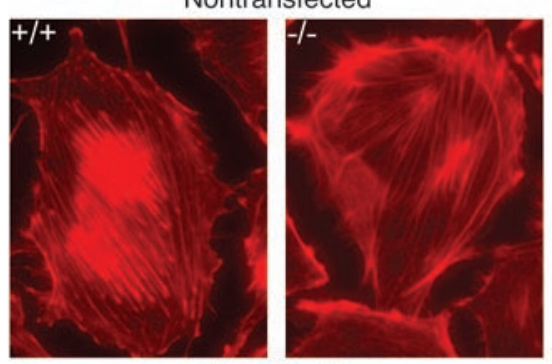

Psuper-synpo

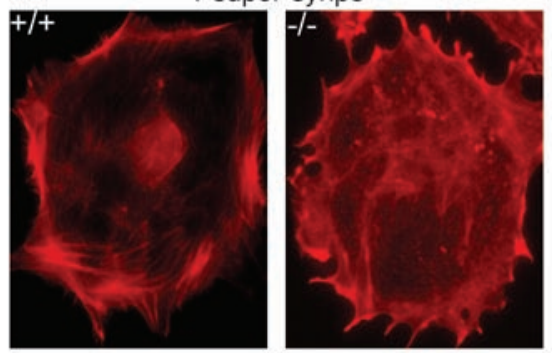

Figure 6

Synpo-T serves as a backup in podocytes but not in brains of synpo-/- mice. (A) Genomic organization and alternative splice products of mouse synaptopodin. In addition to Synpo-long and Synpo-short, a third isoform, Synpo-T, is generated from exon 3. Synpo-T encodes a protein that is identical to AA 721-901 of Synpo-long. E1-E3, exons 1-3. (B) Northern blot analysis showing the induction of Synpo-T mRNA (arrows) in brains $(B)$ and cultured podocytes $(\mathrm{P})$ of synpo-/- mice. In wild-type mice, the Synpo-T probe cross-hybridizes with Synpo-long (arrowhead). (C) Western blot analysis showing the upregulation of Synpo-T protein expression in synpo-/- podocytes. In contrast, Synpo-T is not expressed at the protein level in the brain. (D) Synpo-T is sufficient to convert FLAG- $\alpha$-actinin-4-induced short, branched filaments into long, parallel, unbranched actin bundles (magnification, $\times 450$ ). (E) Western blot analysis of Synpo-long and Synpo-T before (nontransfected) and after gene silencing by stable expression of Synpo-long- and Synpo-T-specific siRNA (Psuper-synpo). Psuper-synpo but not a control siRNA construct (Psuper-control) abrogates Synpo-long and Synpo-T protein expression in wild-type and synpo-/- podocytes. Equal protein loading was assessed by reprobing for tubulin. (F) Phalloidin staining reveals well-developed stress fibers in nontransfected wild-type and synpo-/- podocytes. In contrast, wild-type and synpo-/- podocytes expressing Psuper-synpo are devoid of stress fibers (magnification, $\times 650$ ).

in forebrains of wild-type but not synpo-/- mice. Glomerular $\alpha$ actinin-4 levels were comparable in wild-type and synpo-/- mice at 2 (data not shown) and 6 months of age (Figure 4F). Forebrain $\alpha$-actinin- 2 levels were comparable in wild-type and synpo ${ }^{-/-}$mice at 2 months of age (data not shown). In contrast, $\alpha$-actinin- 2 expression was downregulated in forebrains of 6-month-old synpo-/- mice compared with wild-type littermates (Figure 4F). Equal protein load in all lanes was confirmed by reprobing for tubulin (Figure 4F). These data suggest that the absence of Synpo-short and Synpo-long differentially affects the expression or stability of $\alpha$-actinin isoforms.

$\alpha$-Actinin but not synaptopodin induces short, branched actin filaments. To delineate the functional relevance of the synaptopodin$\alpha$-actinin interaction, we analyzed the actin cytoskeleton in single and cotransfection studies of HEK cells. HEK cells are devoid of stress fibers and express virtually no endogenous synaptopodin or $\alpha$-actinin (data not shown). Therefore, HEK cells are a good model system in which to explore the morphogenic activity of synaptopodin and $\alpha$-actinin. Single transfection of GFP-Synposhort or GFP-Synpo-long resulted in induction of and colocalization with phalloidin-positive amorphous actin aggregates, suggesting the presence of actin binding sites (Figure 5, A and B). In contrast, GFP-Synpo-alt was distributed throughout the cell but did not induce phalloidin-positive aggregates (Figure 5C). Single transfection of GFP- $\alpha$-actinin- 4 induced the formation of short, branched actin filament bundles (Figure 5D).

Synaptopodin bundles and elongates $\alpha$-actinin-induced actin filaments in an isoform-specific manner. Next we performed cotransfections of GFP-Synpo-short, GFP-Synpo-long, or GFP-Synpo-alt with FLAG- $\alpha$-actinin- 4 and analyzed the effects on the actin cytoskeleton (Figure 5, E and F). GFP-Synpo-short (Figure 5E), GFPSynpo-alt (Figure 5F), and GFP-Synpo-long (data not shown) induced the bundling and elongation of the $\alpha$-actinin-4-induced short, branched actin filaments, albeit with different kinetics (see 


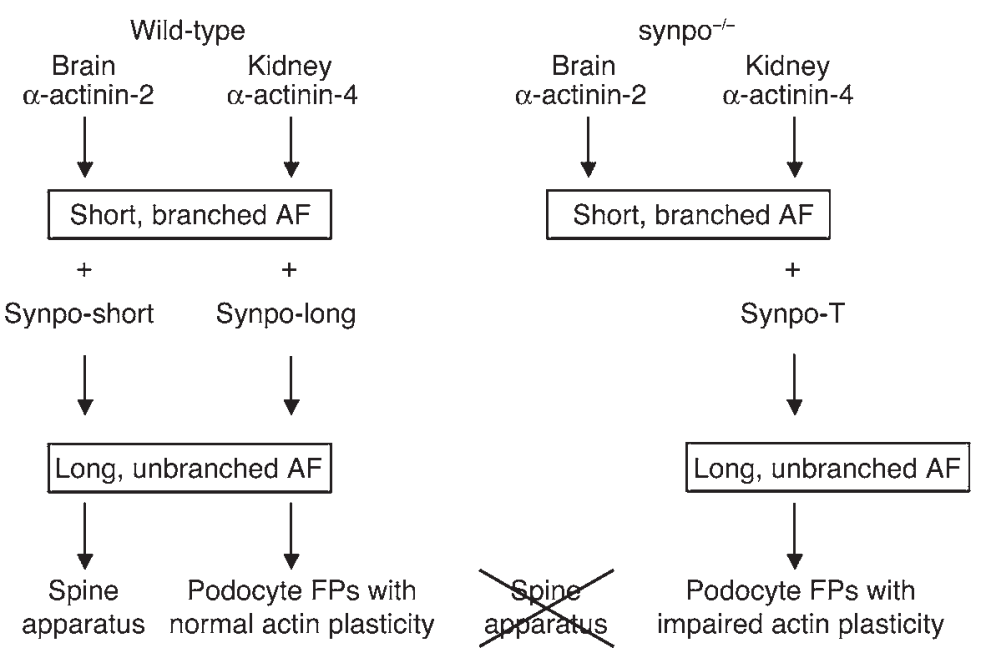

\begin{abstract}
Figure 7
Working model of the cellular function of synaptopodin. In the wild-type brain, Synpo-short converts $\alpha$-actinin2-induced short, branched actin filaments (AF) into long, unbranched actin filaments that are found between the ER stacks of the spine apparatus. Similarly, Synpo-long converts short, cortical $\alpha$-actinin-induced actin filaments of immature podocytes into long, unbranched contractile filaments found in FPs of differentiated podocytes. In the brains of synpo-/- mice, Synpo-T is not expressed, leading to the loss of the spine apparatus. In contrast, Synpo-T is upregulated in synpo-/- podocytes and can partially rescue the loss of Synpo-long in podocytes, albeit at the price of a delayed plasticity of the actin filaments.
\end{abstract}

below, Figure 5I). In the dendritic spine apparatus of wild-type mice, Synpo-short is coexpressed with $\alpha$-actinin-2 (Figure 4D). To test whether the effects of synaptopodin were specific for $\alpha$-actinin-4, we repeated the cotransfections of GFP-Synpo-short, GFP-Synpo-alt, or GFP-Synpo-long with FLAG- $\alpha$-actinin-2 (Figure 5, G and H). Cotransfection of GFP-Synpo-short (Figure 5G) or GFP-Synpo-long (data not shown) with $\alpha$-actinin-2 induced actin filaments that were indistinguishable from those induced by cotransfection with FLAG- $\alpha$-actinin-4 (Figure 5, E and F). In contrast, GFP-Synpo-alt showed isoform specificity for $\alpha$-actinin-4, because we never observed the elongation and bundling of actin filaments in cells cotransfected with GFP-Synpo-alt and FLAG- $\alpha$-actinin-2 (Figure $5 \mathrm{H}$ ). To confirm these results in a quantitative fashion, we counted the number of actin bundles in cells cotransfected with FLAG- $\alpha$-actinin-4 (Figure 5I) or FLAG$\alpha$-actinin-2 (Figure 5J) and any of the 3 synaptopodin constructs. At 72 Hours, virtually all cells cotransfected with GFP-Synpo-long and $\alpha$-actinin- $4(98.3 \% \pm 1.31 \%)$ or $\alpha$-actinin- $2(98.4 \% \pm 0.96 \%)$ had developed actin bundles. GFP-Synpo-short did not show significant differences compared with GFP-Synpo-long for cells cotransfected with FLAG- $\alpha$-actinin-2 (98.13\% $\pm 1.80 \%$ for Synposhort versus $98.64 \% \pm 0.96$ for Synpo-long; Figure 5J), whereas after cotransfection with FLAG- $\alpha$-actinin- 4 , only $63.78 \% \pm 5.35 \%$ of cells transfected with GFP-Synpo-short developed any actin bundles at 72 hours (Figure 5I). Similarly, cells coexpressing GFPSynpo-alt and FLAG- $\alpha$-actinin- 4 showed slower kinetics of actin bundling at 24, 48, and 72 hours compared with GPF-Synpo-long (at 72 hours, $50.81 \% \pm 7.98 \%$ for Synpo-alt versus $98.64 \% \pm 0.96 \%$ for Synpo-long) but not compared with GFP-Synpo-short (at 72 hours, $63.78 \% \pm 5.35 \%$ ). In sharp contrast, we did not observe actin bundles in cells cotransfected with GFP-Synpo-alt and $\alpha$-actinin- 2 at any time point analyzed (at 24 hours, $2.86 \% \pm 1.13 \%$; at 48 hours, $4.80 \% \pm 1.995 \%$; at 72 hours, $1.52 \% \pm 1.46 \%$ ).

Synaptopodin induces stress fibers in podocytes. Next, the relevance of the heterologous cell results for podocyte biology was assessed in wild-type podocytes maintained under permissive conditions. Under these conditions, podocytes display a cortical actin cytoskeleton and lack synaptopodin (20). Transfection with GFP-Synpo-long induced the formation of stress fibers (Figure 5K), similar to those observed in differentiated wildtype podocytes (Figure 4B). These results establish the in vivo relevance of the findings in the heterologous HEK 293 cell studies for podocyte biology.

Synpo-T, a truncated synaptopodin isoform, is upregulated in synpo-/podocytes. In contrast to neurons (5), podocytes of synpo-/- mice do not show an overtly abnormal phenotype but present with a delayed restoration of actin filaments (Figure 1). The slower kinetics of actin recovery after cytochalasin D (Figure 1, E and F) is reminiscent of the slower actin-bundling kinetics of Synpoalt compared with Synpo-long (Figure 5I). These data raise the possibility that a Synpo-alt-like protein serves as a backup in synpo-/- podocytes. Rescreening of the NCBI GenBank database identified a mouse cDNA clone (GenBank accession number AK034012) that encodes 2,549 bp including an open reading frame (ORF) with an ATG corresponding to the $\mathrm{C}$ terminus (AA 721-901) of Synpo-long (Figure 6A). We designated this new isoform Synpo-T (Synpo-truncated). By RT-PCR (data not shown) and Northern blot analysis, Synpo-T mRNA was detected in brain and cultured podocytes of synpo-/- but not wild-type mice (Figure 6B). Instead, as expected, in wild-type podocytes the Synpo-T probe detected the Synpo-long mRNA (Figure 6B). Next, we raised a Synpo-T specific polyclonal antibody and explored the protein expression of Synpo-T by Western blotting (Figure 6C). Synpo-T protein expression could not be detected in brain extracts of wild-type or synpo-/- mice (Figure 6C, left). In contrast, Synpo-T protein expression was upregulated in synpo- ${ }^{-/}$ podocytes compared with wild-type cells (Figure 6C, right). Because Synpo-T is a truncated version of Synpo-alt, we tested the ability of Synpo-T to bundle and elongate $\alpha$-actinin-4induced actin filaments. When GFP-Synpo-T was cotransfected with FLAG-actinin-4, it had the same capability to bundle and elongate $\alpha$-actinin-4-induced actin filaments as did GFP-Synpoalt (Figure 6D). These data suggest that Synpo-T serves as functional backup in podocytes of synpo-/- null mice.

Gene silencing of Synpo-long and Synpo-T abrogates stress fiber formation in podocytes. To explore further the mechanistic role of Synpo-long and Synpo-T in actin filament dynamics, we took a loss-of-function approach by small interfering RNAs (siRNAs) (21). After inactivation of Synpo-long and Synpo-T in wild-type cells, and Synpo-T in synpo-/- cells, by stable expression of a siRNA that can silence both Synpolong and Synpo-T, we observed the loss of synaptopodin pro- 
tein expression in wild-type and synpo-/- podocytes (Figure 6E). These changes were specifically due to the ablation of synaptopodin expression, because they were not seen in cells transfected with a mutated control siRNA (Figure 6E). Functionally, the suppression of synaptopodin expression caused the loss of stress fibers, both in wild-type and synpo-/- cells (Figure 6F). Together with the gain-of-function studies described above (Figure $5 \mathrm{~K}$ ), these data show that synaptopodin is necessary and sufficient to induce stress fibers in podocytes.

\section{Discussion}

So far, the best-defined function of $\alpha$-actinin is to cross-link and bundle actin filaments (10). In this study, we have discovered that synaptopodin can bind to $\alpha$-actinin and inhibit, in an isoformspecific fashion, the branching of $\alpha$-actinin-induced actin filaments, thereby causing the bundling and elongation of these filaments. This study also shows that the lack of Synpo-short leads to a time-dependent reduction of $\alpha$-actinin- 2 expression in the brain but not of $\alpha$-actinin- 4 in the kidney. The difference in the function of the interaction of $\alpha$-actinin- 2 versus $\alpha$-actinin- 4 with synaptopodin is, to our best knowledge, the first clear example of a biochemical difference between these $\alpha$-actinin isoforms. Moreover, we established that synpo-/- podocytes upregulate Synpo-T, a truncated isoform that can partially rescue the functionality of synaptopodin's interaction with $\alpha$-actinin- 4 but not $\alpha$-actinin- 2 . We also found that the expression of synaptopodin induces stress fibers in podocytes and that gene silencing of synaptopodin abrogates stress fiber formation in podocytes. In concert, these data can explain why synpo-/- mice show an overtly abnormal phenotype in the brain, where Synpo-short interacts with $\alpha$-actinin-2, and not in the kidney, where Synpo-long and Synpo-T interact with $\alpha$-actinin-4 (Figure 7).

The eukaryotic actin cytoskeleton is organized into 2 major forms. In the submembranous region, a network of short, branched filaments is present; deeper in the cortex, in stress fibers, microvilli, and muscle sarcomeres, actin filaments are much longer and virtually not branched (22-24). The underpinning mechanism governing actin polymerization and filament branching are now well understood (25). Much less is known about the mechanisms regulating filament elongation and generation of parallel actin bundles (26). One protein family that causes the elongation of microvillus-type parallel actin bundles in vivo is the espins (27). Interestingly, espins are found in the cerebellum but not in the forebrain (28), where synaptopodin is expressed (2).

How might synaptopodin modulate the actin bundling of $\alpha$-actinin? Some time ago, the physical nature of $\alpha$-actininactin networks was shown to depend on the length of the actin filaments, the affinity of $\alpha$-actinin for actin, and the concentrations of actin and $\alpha$-actinin (29). Pollard and colleagues showed the capacity of $\alpha$-actinin and actin to form isotropic networks, rather than bundles, and defined the concept of threshold for bundling that depends on the affinity of $\alpha$-actinin for actin (29). This is in line with another, more recent, in vitro study showing that when complexed with $\alpha$-actinin, F-actin will spontaneously assemble into relatively rigid, frequently branching, networks of actin bundles (30). These data raise the intriguing possibility that other proteins such as synaptopodin alter the affinity or avidity of $\alpha$-actinin for actin, thereby inducing the formation of long, unbranched, parallel bundles.
In summary, in the absence of synaptopodin, $\alpha$-actinin induces short, branched actin filaments (Figure 7). In vivo, such branched filaments are found in immature podocytes that lack FPs and show a typical cortical actin filament system (18). In the presence of synaptopodin, the branching activity of $\alpha$-actinin is blocked, and unbranched parallel actin bundles develop. In vivo, as shown here and before (1), the expression of synaptopodin coincides with the formation of podocyte FPs and the development of their parallel contractile actin bundles typical of differentiated adult podocytes (19), suggesting that synaptopodin induces the typically long, unbranched actin filaments of differentiated podocytes. Similarly, in the brain, synaptopodin may induce the long, unbranched actin filaments that are found in the dense plates between the stacks of the dendritic spine apparatus (31). Thus, our studies have helped to define a mechanistic role for synaptopodin as a regulator of the actin-bundling activity of $\alpha$-actinin that may be important for the formation and dynamic reorganization of the actin cytoskeleton in highly specialized cell compartments, such as podocyte FPs and the spine apparatus in the brain.

\section{Methods}

Transmission electron microscopic morphology. All animal studies reported here were approved by the Albert Einstein College of Medicine Animal Institute

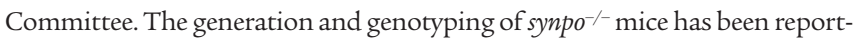
ed previously (5). All studies were done in a pure 129 background. Kidney morphology was analyzed according to our published protocols (32).

PS-induced FP effacement. Adult wild-type $(n=8)$ and synpo-/- $(n=7)$ littermate mice were anesthetized with pentobarbital and both kidneys were perfused in situ through the left ventricle at a pressure of approximately $120 \mathrm{mmHg}$ and an infusion rate of $9 \mathrm{ml} / \mathrm{min}$ (14). The kidneys remained immersed in a water bath at $37^{\circ} \mathrm{C}$ during the entire experiment. First, kidneys were flushed with HBSS at $37^{\circ} \mathrm{C}$ for 2 minutes, followed by perfusion with PS $(2 \mathrm{mg} / \mathrm{ml}$ in HBSS) at $37^{\circ} \mathrm{C}$ for 15 minutes. For the reperfusion studies, the animals were perfused with heparin $(800 \mu \mathrm{g} / \mathrm{ml}$ in HBSS $)$ at $37^{\circ} \mathrm{C}$ for 15 minutes after perfusion with PS (33). At the end of the experiments, the kidneys were fixed by perfusion with $4 \%$ paraformaldehyde in PBS, removed, and immersed in the same fixative for a maximum of 2 days until further processing for transmission electron microscopy (TEM). The degree of FP effacement was assessed in a series of 20 pictures per animal taken at $\times 15,000$ magnification. The degree of FP effacement was determined as a percentage using the following formula: GBM surface covered by FP/total GBM surface $\times 100$. Results are expressed as mean \pm SD and assessed by Student's $t$ test and ANOVA.

LPS-induced nephrotic syndrome. Transient nephrotic syndrome with podocyte FP effacement was induced by LPS injection as previously described (15). Baseline urinary protein excretion of 6-week-old female wild-type $(n=10)$ and $\operatorname{synpo}^{-/-}(n=10)$ littermate mice was analyzed by urine dip stick (Albustix; Bayer) before animals were injected intraperitoneally with $200 \mu \mathrm{g}$ LPS $(1 \mathrm{mg} / \mathrm{ml}$ in sterile LPS-free PBS) in a total volume of $200 \mu \mathrm{l}$ (15). To determine the time course of nephrotic syndrome, urinary protein excretion was measured again at various time points after LPS injection $(12,24,36,46,60,72$, and 84 hours).

Generation of Synpo-alt and Synpo-T specific polyclonal antibodies. Rabbits were immunized with a peptide conjugated with keyhole limpet hemocyanin (KLH) corresponding to AA 694-712 of Synpo-long (single-letter code RPPSSLDGWVSPGPWEPGR) that is specific for Synpo-alt, and the antibody was affinity-purified as previously described (2). A Synpo-T specific chicken antiserum was raised by immunization with a KLH-conjugated peptide corresponding to AA 129-145 of mouse Synpo-T (YR RSPTDSD VSLDSEDS) and affinity-purified. This polyclonal chicken anti-Synpo-T IgY recognized a GFP-Synpo-T fusion protein by Western blotting (data not shown). 
Plasmid constructs. PCR products were ligated into pEGFP-C1 (BD Biosciences - Clontech) and pFLAG 5a (Sigma-Aldrich). Plasmids containing $\alpha$-actinin- 2 and $\alpha$-actinin- 4 were provided by M. Pollak (Brigham and Women's Hospital, Boston, Massachusetts, USA) (11). Truncated cDNA constructs encoding Synpo-short fragments SP1 (AA 1-300), SP2 (AA 250-550), SP3 (AA 500-691), and SP3- (AA 550-691) were amplified by PCR from GFP-Synposhort. cDNA constructs encoding Synpo-alt fragments SP4 (AA 1-159), SP5 (AA 160-227), SP6 (AA 1-181), SP7 (AA 103-181), SP8 (AA 103-227), SP9 (AA 182-227), and SP10 (AA 1-102) were amplified from GFP-Synpo-alt by PCR. They were inserted into pEGFP-C1 vector. Full-length $\alpha$-actinin- 4 was amplified by PCR from $\mathrm{PGFP}-\alpha$-actinin- 4 and inserted into PFLAG-CMV-5a vector. All constructs were verified by DNA sequencing.

Yeast 2-bybrid screen. Synpo-alt was cloned into the bait vector pGBKT7 (BD Biosciences - Clontech) in order to create a fusion protein with the GAL4 DNA binding domain and transformed into the yeast strain AH109. A pretransformed human kidney MATCHMAKER cDNA library was screened in accordance with the manufacturer's protocol (MATCHMAKER TwoHybrid System 3; BD Biosciences - Clontech). Prey plasmids were isolated, sequenced, and retransformed into AH109 cells in combination with the Synpo-alt bait construct, a control plasmid (pGBKT7-lamin; BD Biosciences - Clontech), or the empty bait vector pGBKT7 to exclude false positives.

Cell culture and transient transfection. Wild-type podocytes were cultured as previously described (20). synpo-/- conditionally immortalized podocyte cell lines were generated by intercrossing syn $\mathrm{po}^{-/-}$mice with the ImmortoMouse (Charles River) carrying a temperature-sensitive T-antigen as transgene (-). Glomeruli were isolated from kidneys of 6-week-old synpo-1-/T-antigen transgenic mice, and 5 synpo-/- clonal podocyte cell lines were obtained by limiting dilution as previously described for wild-type cells (20). Transient transfections of HEK293 cells (ATCC) were performed using FuGene 6 Reagent (Roche Diagnostics Corp.) at a 1:3 DNA:Fugene ratio in accordance with the manufacturer's protocol. GFP fusion proteins were analyzed by direct fluorescence microscopy in living cells.

Actin depolymerization and recovery studies. Differentiated wild-type and synpo-/- podocytes were treated overnight with $2 \mu \mathrm{M}$ cytochalasin D (SigmaAldrich) to disrupt the actin cytoskeleton (2). Cells were washed thoroughly to remove the cytochalasin D and further maintained in regular RPMI 1640 medium supplemented with $10 \%$ FCS. The recovery of the actin cytoskeleton was monitored at defined time points ( $0,3,6,12,24$, and 48 hours). Immunofluorescence microscopy was done as previously described (3). F-actin was visualized with rhodamine-labeled phalloidin (Invitrogen Corp.). Interference reflection microscopy was performed with a Radiance 2000 confocal microscope (Bio-Rad Laboratories) in reflectance mode with polarization filters at either 488 or $568 \mathrm{~nm}$. Areas of actin fibers were identified via rhodamine phalloidin staining and converted to black pixels. The content of F-actin was determined by a software program, which allowed us to measure simultaneously the number of actin stress fibers per cell and the individual cell size. The ratio of stress fibers to total cell size was quantified using NIH Image software (http://www.cc.nih.gov/cip/ip_packages/ip_packages.html).

Tissue extraction, Western blotting, and immunoprecipitation. Protein extraction from brains and isolated kidney glomeruli, SDS-PAGE, and Western blotting were done as previously described (2). For coimmunoprecipitation of FLAG and GFP fusion proteins, HEK293 cells were grown on a $6-\mathrm{cm}$ dish, cotransfected at a confluence of approximately $75 \%$ and harvested on ice after 24 hours using $5 \mathrm{ml}$ of PBS in $50 \mathrm{mM}$ EDTA. Cells were pelleted by centrifugation at $800 \mathrm{~g}$ for 5 minutes at $4^{\circ} \mathrm{C}$ and washed twice with ice-cold PBS. For cell lysis, the pellet was resuspended in $1 \mathrm{ml}$ IP buffer $(50 \mathrm{mM}$ Tris [ $\mathrm{pH} 7.5$ ], $150 \mathrm{mM} \mathrm{NaCl}, 1.0 \%$ Triton X100, and protease inhibitors) and incubated on ice for 30 minutes. The cell lysate was cleared by centrifugation for 5 minutes at $18,000 \mathrm{~g}$. One milliliter of cell extract was incubated with $40 \mu$ lagarose beads coated with anti-FLAG-M2 antibody (SigmaAldrich) at $4^{\circ} \mathrm{C}$ for 2 hours. Beads were collected by centrifugation for 1 minute at $1,000 \mathrm{~g}$ and washed 5 times with $1 \mathrm{ml}$ of IP buffer for $30 \mathrm{~min}$ on a rotator. Bound proteins were eluted by boiling agarose beads in $50 \mu \mathrm{l} \mathrm{Laemmli} \mathrm{buffer}$ at $95^{\circ} \mathrm{C}$ for 5 minutes and analyzed by immunoblotting using an anti-GFP antibody. Antibodies against FLAG and GFP were used at 1:10,000 and 1:300, respectively. Anti- $\alpha$-actinin (sarcomeric; Sigma-Aldrich) and anti- $\alpha$-actinin-4 (11) were used at $1: 4,000$ and 1:5,000, respectively. Synaptopodin NT (2) and anti-Synpolong antibodies were used at 1:300. HRP-conjugated secondary antibodies (Promega Corp.) were used at 1:10,000. The immunoreaction was visualized with ECL or ECL plus (Amersham Pharmacia Biotech). Endogenous coimmunoprecipitations of lysates from differentiated wild-type podocytes were done as previously described (35) using the following rabbit polyclonal primary antibodies: anti-Synpo-alt, anti$\alpha$-actinin-4, and anti-WT-1 (negative control).

RT-PCR and Northern blotting. RT-PCR for mouse brain and isolated glomeruli was performed as previously described (2) using the following primers: Synpo-short (forward, GCCTGCCTCTCTCTACCACGG; reverse, GAAGCAGAAGGAAGGCTTCCACAC) yielding a 475-bp product; Synpo-long (forward, GCCTGCCTCTCTCTACCACGG; reverse, GAAGCAGAAGGAAGGCTTCCACAC) yielding a 618-bp product. For Northern blots, total RNA was harvested from mouse brains and cultured podocytes of wild-type and synpo-/- mice using TRIzol (GIBCO Life Technology). A ${ }^{32} \mathrm{P}-$ labeled Synpo-T- and Synpo-long-specific cDNA probe was generated with the Random Primed DNA Labeling Kit (Boehringer Mannheim). The blots were hybridized at $68^{\circ} \mathrm{C}$ overnight in MiracleHyb hybridization solution (Stratagene) in accordance with the manufacturer's instructions and visualized by autoradiography.

siRNA. The plasmid vector pSUPER (Oligoengine) (21) was used for stable expression of siRNA in wild-type and synpo- $\mathrm{o}^{--}$podocytes. The synaptopodin specific insert was a 19 -nt sequence (TGTCCCCCTCGTGGAGCGA) corresponding to nt 38-56 of the mouse Synpo-T ORF, which is separated by a 9 -nt noncomplementary spacer (TTCAAGAGA) from the reverse complement of the same 19-nt sequence. A control vector (pSUPER-control) was constructed using a 19-nt scrambled sequence (CCGCGACTCGCCGTCTGCG) with no significant homology to any mammalian gene sequence. These sequences were inserted into PSUPER after digestion with BglII and HindIII (Promega Corp.). The insertion of the oligonucleotide sequence was verified by DNA sequencing. The zeocin gene was amplified by PCR from pcDNA3.1/Zeo (Invitrogen Corp.) and subcloned into pSUPER. Podocytes were transfected with $1 \mu \mathrm{g}$ of siRNA plasmid using FuGENE transfection agent (Roche) in accordance with the manufacturer's instructions. Seventy-two hours after transfection, stable transfectants were selected with $400 \mu \mathrm{g} / \mathrm{ml}$ zeocin (Invitrogen Corp.). Clonal cell lines were selected and tested for Synpo-long and Synpo-T expression by Western blotting as described above.

\section{Acknowledgments}

We thank Svetlana Ratner, Mary Donnelly, and Anthony Visioni for excellent technical assistance, as well as Michael Cammer (Albert Einstein College of Medicine) for help with F-actin quantification. We thank Martin Pollak (Brigham and Women's Hospital, Boston, Massachusetts, USA) for $\alpha$-actinin cDNA constructs and antibodies. It is a great pleasure to acknowledge the long-term support of this project by Wilhem Kriz (University of Heidelberg, Heidelberg, Germany), as well as many helpful discussions with John Condeelis (Albert Einstein College of Medicine). We also thank Dontscho Kerjaschki (Medical University of Vienna, Vienna, Austria) for advice on the PS/heparin experi- 
ments. K. Asanuma was supported by the Kidney and Urology Foundation of America (KUFA), the National Kidney Foundation, the Alumni Association of Juntendo University (Tokyo, Japan), and a personal grant from Yasuhiko Tomino (Juntendo University, Tokyo, Japan). K. Kim was supported by KUFA, and J. Oh was supported by KUFA and the Deutsche Forschungsgemeinschaft. This work was supported by NIH grants DA18886, DK57683, and DK062472, and the George M. O'Brien Kidney Center grant DK064236.

Received for publication September 16, 2004, and accepted in revised form February 8, 2005.

Address correspondence to: P. Mundel, Division of Nephrology, Mount Sinai School of Medicine, One Gustave L. Levy Place, Box 1243, New York, New York 10029-6574, USA. Phone: (212) 659-9332; Fax: (212) 849-2643; E-mail: peter.mundel@mssm.edu.
Katsuhiko Asanuma, Kwanghee Kim, Christian Faul, and Peter Mundel's present address is: Department of Medicine, Mount Sinai School of Medicine, New York, New York, USA.

Laura Giardino's present address is: Renal Research Association, Renal Immunopathology Center, San Carlo Borromeo Hospital, Milan, Italy.

Jochen Reiser's present address is: Renal Unit, Massachusetts General Hospital, and Harvard Medical School, Charlestown, Massachusetts, USA.

Jun Oh's present address is: Children's Hospital, University of Heidelberg, Heidelberg, Germany.

Katsuhiko Asanuma, Kwanghee Kim, and Jun Oh contributed equally to this work.
1. Mundel, P., Gilbert, P., and Kriz, W. 1991. Podocytes in glomerulus of rat kidney express a characteristic $44 \mathrm{KD}$ protein. J. Histochem. Cytochem. 39:1047-1056.

2. Mundel, P., et al. 1997. Synaptopodin: an actinassociated protein in telencephalic dendrites and renal podocytes. J. Cell Biol. 139:193-204.

3. Weins, A., et al. 2001. Differentiation- and stressdependent nuclear cytoplasmic redistribution of myopodin, a novel actin-bundling protein. J. Cell Biol. 155:393-404.

4. Deller, T., Merten, T., Roth, S.U., Mundel, P., and Frotscher, M. 2000. Actin-associated protein synaptopodin in the rat hippocampal formation: localization in the spine neck and close association with the spine apparatus of principal neurons. J. Comp. Neurol. 418:164-181.

5. Deller, T., et al. 2003. Synaptopodin-deficient mice lack a spine apparatus and show deficits in synaptic plasticity. Proc. Natl. Acad. Sci. U. S. A 100:10494-10499.

6. Blanchard, A., Ohanian, V., and Critchley, D. 1989. The structure and function of alpha-actinin. J. Muscle Res. Cell Motil. 10:280-289.

7. Pascual, J., Castresana, J., and Saraste, M. 1997. Evolution of the spectrin repeat. Bioessays. 19:811-817.

8. Beggs, A.H., et al. 1992. Cloning and characterization of two human skeletal muscle alpha-actinin genes located on chromosomes 1 and 11. J. Biol. Chem. 267:9281-9288.

9. Djinovic-Carugo, K., Young, P., Gautel, M., and Saraste, M. 1999. Structure of the alpha-actinin rod: molecular basis for cross-linking of actin filaments. Cell. 98:537-546.

10. Otey, C.A., and Carpen, O. 2004. Alpha-actinin revisited: A fresh look at an old player. Cell Motil. Cytoskeleton. 58:104-111.

11. Kaplan, J.M., et al. 2000. Mutations in ACTN4, encoding alpha-actinin-4, cause familial focal segmental glomerulosclerosis. Nat. Genet. 24:251-256.

12. Yao, J., et al. 2004. Alpha-actinin-4-mediated FSGS: an inherited kidney disease caused by an aggregated and rapidly degraded cytoskeletal protein. PLoS Biol. 2:787-794.
13. Seiler, M.W., Venkatachalam, M.A., and Cotran, R.S. 1975. Glomerular epithelium: structural alterations induced by polycations. Science. 189:390-393.

14. Kerjaschki, D. 1978. Polycation-induced dislocation of slit diaphragms and formation of cell junctions in rat kindey glomeruli. The effects of low temperature, divalent cations, colchicine, and cytochalasin B. Lab. Invest. 39:430-440.

15. Reiser, J., et al. 2004. Induction of B7-1 in podocytes is associated with nephrotic syndrome. J. Clin. Invest. 113:1390-1397. doi:10.1172/JCI200420402.

16. Renfranz, P.J., and Beckerle, M.C. 2002. Doing (F/L)PPPPs: EVH1 domains and their proline-rich partners in cell polarity and migration. Curr. Opin. Cell Biol. 14:88-103.

17. Wyszynski, M., et al. 1998. Differential regional expression and ultrastructural localization of alpha-actinin-2, a putative NMDA receptor-anchoring protein, in rat brain. J. Neurosci. 18:1383-1392.

18. Mundel, P., and Kriz, W. 1995. Structure and function of podocytes: an update. Anat. Embryol. 192:385-397.

19. Drenckhahn, D., and Franke, R.P. 1988. Ultrastructural organization of contractile and cytoskeletal proteins in glomerular podocytes of chicken, rat, and man. Lab. Invest. 59:673-682.

20. Mundel, P., et al. 1997. Rearrangements of the cytoskeleton and cell contacts induce process formation during differentiation of conditionally immortalized mouse podocyte cell lines. Exp. Cell Res. 236:248-258.

21. Brummelkamp, T.R., Bernards, R., and Agami, R. 2002. A system for stable expression of short interfering RNAs in mammalian cells. Science. 296:550-553.

22. Small, J.V. 1995. Structure-function relationships in smooth muscle: the missing links. Bioessays. 17:785-792.

23. Bailly, M., et al. 1999. Relationship between Arp2/3 complex and the barbed ends of actin filaments at the leading edge of carcinoma cells after epidermal growth factor stimulation. J. Cell Biol. 145:331-345.

24. Svitkina, T.M., and Borisy, G.G. 1999. Progress in protrusion: the tell-tale scar. Trends Biochem. Sci.
24:432-436.

25. Pollard, T.D., and Borisy, G.G. 2003. Cellular motility driven by assembly and disassembly of actin filaments. Cell. 112:453-465.

26. Bartles, J.R. 2000. Parallel actin bundles and their multiple actin-bundling proteins. Curr. Opin. Cell Biol. 12:72-78.

27. Loomis, P.A., et al. 2003. Espin cross-links cause the elongation of microvillus-type parallel actin bundles in vivo. J. Cell Biol. 163:1045-1055.

28. Sekerkova, G., et al. 2003. Novel espin actinbundling proteins are localized to Purkinje cell dendritic spines and bind the Src homology 3 adapter protein insulin receptor substrate p53. J. Neurosci. 23:1310-1319.

29. Wachsstock, D.H., Schwartz, W.H., and Pollard, T.D. 1993. Affinity of alpha-actinin for actin determines the structure and mechanical properties of actin filament gels. Biophys. J. 65:205-214.

30. Pelletier, O., et al. 2003. Structure of actin crosslinked with alpha-actinin: a network of bundles. Phys. Rev. Lett. 91:148102.

31. Capani, F., Martone, M.E., Deerinck, T.J., and Ellisman, M.H. 2001. Selective localization of high concentrations of F-actin in subpopulations of dendritic spines in rat central nervous system: a three-dimensional electron microscopic study. J. Comp. Neurol. 435:156-170.

32. Mundel, P., Elger, M., Sakai, T., and Kriz, W. 1988. Microfibrils are a major component of the mesangial matrix in the glomerulus of the rat kidney. Cell Tissue Res. 254:183-187.

33. Seiler, M.W., Rennke, H.G., Venkatachalam, M.A., and Cotran, R.S. 1977. Pathogenesis of polycationinduced alterations ("fusion") of glomerular epithelium. Lab. Invest. 36:48-61.

34. Jat, P.S., et al. 1991. Direct derivation of conditionally immortal cell lines from an $\mathrm{H}-2 \mathrm{~Kb}-\mathrm{ts} \mathrm{A} 58$ transgenic mouse. Proc. Natl. Acad. Sci. U. S. A. 88:5096-5100.

35. Schwarz, K., et al. 2001. Podocin, a raft-associated component of the glomerular slit diaphragm, interacts with CD2AP and nephrin. J. Clin. Invest. 108:1621-1629. doi:10.1172/JCI200112849. 